\title{
An Optimized Most Probable Number (MPN) Method to Assess the Number of Thermophilic Free-Living Amoebae (FLA) in Water Samples
}

\author{
Mirna Moussa ${ }^{1}$, Isabel Marcelino ${ }^{1, *}$, Vincent Richard ${ }^{2}$, Jérôme Guerlotté ${ }^{3}{ }^{\circledR}$ and \\ Antoine Talarmin ${ }^{1}$ \\ 1 Unité TReD-Path (Transmission Réservoir \& Diversité des Pathogènes), Institut Pasteur de la Guadeloupe, \\ Les Abymes, 97183 Guadeloupe, France; mirna.moussa.83@gmail.com (M.M.); \\ ATALARMIN@pasteur-guadeloupe.fr (A.T.) \\ 2 Institut Pasteur, 75015 Paris, France; vincent.richard@pasteur.fr \\ 3 Institut de Systématique, Evolution, Biodiversité (ISYEB) MNHN, CNRS, Sorbonne Université, \\ EPHE Université des Antilles, Pointe-à-Pitre, 97110 Guadeloupe, France; jerome.guerlotte@univ-antilles.fr \\ * Correspondence: IMarcelino@pasteur-guadeloupe.fr; Tel.: +590-590-897-664
}

Received: 6 April 2020; Accepted: 1 May 2020; Published: 24 May 2020

check for updates

\begin{abstract}
Detection and quantification of pathogenic free-living amoebae (FLA) in water samples is critical for assessing water quality and for disease management issues. The most probable number (MPN) is commonly used to account for FLA in water. Nevertheless, this requires a high number of water replicates and working volumes, and a consequent number of non-nutrient agar (NNA)-plates seeded with Escherichia coli. Herein, we aimed at optimizing this difficult method, taking also into account key factors such as (i) the counting method, (ii) the delay between sample collection and sample processing, and (iii) the temperature during water sample transportation. To simplify the MPN method, we filtrated $1 \times 1000$ and $1 \times 100 \mathrm{~mL}$ water samples, and cellulose acetate filters were cut in 10 parts and inverted on NNA-plates overlaid with E. coli. The comparison between the classical and our optimized MPN method showed that the final counts were similar, therefore validating the use of the optimized method. Our results also showed that for thermophilic FLA (such as Naegleria fowleri), water samples can be kept at around $+30^{\circ} \mathrm{C}$ and processed within $24 \mathrm{~h}$. This improved MPN method is now routinely used in our laboratory to control Naegleria sp. in the water samples in Guadeloupe.
\end{abstract}

Keywords: free-living amoebae; thermophilic amoebae; Naegleria fowleri; most probable number (MPN); optimized quantification method

\section{Introduction}

Free-living amoebae (FLA) are ubiquitous unicellular organisms, being found in water, soil, dust, and air samples. Some FLA are thermophilic, being able to survive and/or replicate at temperatures equal or above $37^{\circ} \mathrm{C}$ [1-3]. Amongst these, some can be pathogenic to humans and animals such as Acanthamoeba sp., Naegleria sp., and Balamuthia mandrillaris [4,5]. Others are nonpathogenic but of medical importance because they can act as hosts, vehicles, and training grounds for bacteria, such as Vannella sp. [6] and Vermamoeba vermiformis [7-10].

The thermophilic FLA Naegleria fowleri is frequently found in freshwater and soil [11,12], and it may cause primary amoebic meningoencephalitis (PAM), a rare but often fatal disease of the central nervous system [13]. It is generally acquired while swimming or during other recreational activities in freshwater lakes and ponds. Infection of the brain occurs after N. fowleri reaches the nasal cavity 
and invades the nasal mucosa. Afterwards, N. fowleri penetrates the nasal epithelium to the olfactory nerves and migrates through the cribriform plate to invade the brain and meninges [14].

In Guadeloupe (French West Indies), a fatal case of PAM occurred in 2008 after a child swam in a bath fed with geothermal waters [15]. In 2013, our group revealed that thermophilic FLA (and in particular, N. fowleri) were frequently detected in these baths [12]; the contamination of the water with $N$. fowleri occurs after emerging from the geothermal source, when the water runs over the soil [11]. We also observed that $N$. fowleri was often found at low concentrations, below 22 amoebae per liter [12]. These low concentrations in environmental water often make it difficult to accurately count the FLA [16], regardless of the method that is used to concentrate the samples, either by centrifugation or filtration [17]. Because of the risk associated to the presence of $N$. fowleri (the presence of one single amoeba is enough to cause or start an infection) and the potential link between the concentration of $N$. fowleri and pathogenicity, it is important to obtain the most accurate count of this amoeba in water [18-20].

Several methods have been developed to detect and enumerate thermophilic and pathogenic FLA, such as loop-mediated isothermal amplification (LAMP) [21], PCR (conventional and quantitative), and cytometry $[16,17,22-25]$. Still, the most probable number (MPN) method (followed by FLA identification by microscopic observation and/or PCR) is the most commonly used to estimate the concentration of viable microorganisms in a sample by means of replicate amoeba growth in ten-fold dilutions [26-29]. Nevertheless, this is time-consuming and requires a high number of water sample replicates and working volumes and a consequent number of non-nutrient agar (NNA)-plates seeded with E. coli.

Herein, we aimed at optimizing this MPN method by reducing the working volumes and number of replicates. Additionally, we evaluated the impact of the delay between sample collection and sample processing, and the temperature during water sample transportation, on the counts of thermophilic FLA.

\section{Results}

\subsection{Optimization of the MPN Method}

In Table 1, we present the number of thermophilic FLA (sensu lato) and, in particular, for N. fowleri obtained after using the classical (cMPN) and the modified (mMPN) MPN methods. The results show that for FLA, the percentage of samples with equal or higher counts obtained with the mMPN method compared to those obtained with the classic method are $84 \%$ (16/19 samples) for both $100 \mathrm{~mL}$ boxes and $10 \mathrm{~mL}$ boxes. Nevertheless, regardless of the sample, the slight differences are not statistically significant (Mc Nemar Test, $p$-value $=0.48$ for $100 \mathrm{~mL}$ boxes and $p$-value $=0.70$ for $10 \mathrm{~mL}$ boxes) . Moreover, only 2 samples (samples 1 and 13) tested positive for $N$. fowleri using the cMPN, while the optimized method revealed the presence of this amoeba in six water samples (samples 1, 8, 13, 14, 15, and 19).

As we were concerned about accurately assessing the count of pathogenic $N$. fowleri, we also seeded water samples with known quantities of pure $N$. fowleri. For this, we counted the number of positive dilutions (for cMPN) or the number of positive filters (for $\mathrm{mMPN}$ ) for amoeba growth and reported these values to MPN tables [30]. Indeed, using a $100 \mathrm{~mL}$ sample volume, we obtained a higher number of $N$. fowleri colonies with the mMPN compared to the cMPN ( $p=0.03$, Wilcoxon test), although the number of amoebae/L artificially seeded was within the range of counts obtained with both MPN methods. 
Table 1. The number of thermophilic free-living amoeba and Naegleria fowleri colonies observed and the most probable number obtained with the classical most probable number (MPN) method and the modified MPN method.

\begin{tabular}{|c|c|c|c|c|c|c|c|c|c|c|c|c|}
\hline \multirow{4}{*}{ Samples } & \multicolumn{6}{|c|}{ Thermophilic FLA } & \multicolumn{6}{|c|}{ Naegleria fowleri } \\
\hline & \multicolumn{3}{|c|}{ Classical MPN Method } & \multicolumn{3}{|c|}{ Modified MPN Method } & \multicolumn{3}{|c|}{ Classical MPN Method } & \multicolumn{3}{|c|}{ Modified MPN Method } \\
\hline & \multicolumn{2}{|c|}{ Positive Petri Boxes } & \multirow{2}{*}{$\begin{array}{c}\text { MPN } \\
\text { Number } \\
\text { (FLA/L) }\end{array}$} & \multicolumn{2}{|c|}{ Positive Filter Pieces } & \multirow{2}{*}{$\begin{array}{c}\text { MPN } \\
\text { Number } \\
\text { (FLA /L) }\end{array}$} & \multicolumn{2}{|c|}{ Positive Petri Boxes } & \multirow{2}{*}{$\begin{array}{c}\text { MPN } \\
\text { Number } \\
\text { Nf/L }\end{array}$} & \multicolumn{2}{|c|}{ Positive Filter Pieces } & \multirow{2}{*}{$\begin{array}{c}\text { MPN } \\
\text { Number } \\
N f / \mathrm{L}\end{array}$} \\
\hline & $100 \mathrm{~mL}$ & $10 \mathrm{~mL}$ & & $100 \mathrm{~mL}$ & $10 \mathrm{~mL}$ & & $100 \mathrm{~mL}$ & $10 \mathrm{~mL}$ & & $100 \mathrm{~mL}$ & $10 \mathrm{~mL}$ & \\
\hline 1 & 10 & 3 & 86 (39-191) & 9 & 6 & $80(37-175)$ & 3 & 0 & $7(3-20)$ & 1 & 2 & $6(2-19)$ \\
\hline 2 & 3 & 3 & $14(6-31)$ & 5 & 3 & $21(10-43)$ & 0 & 0 & $<2$ & 0 & 0 & $<2$ \\
\hline 3 & 10 & 10 & $>461$ & 10 & 10 & $>461$ & 0 & 0 & $<2$ & 0 & 0 & $<2$ \\
\hline 4 & 1 & 0 & $2(1-14)$ & 1 & 0 & $2(1-14)$ & 0 & 0 & $<2$ & 0 & 0 & $<2$ \\
\hline 5 & 0 & 0 & $<2$ & 0 & 0 & $<2$ & 0 & 0 & $<2$ & 0 & 0 & $<2$ \\
\hline 6 & 10 & 10 & $>461$ & 10 & 10 & $>461$ & 0 & 0 & $<2$ & 0 & 0 & $<2$ \\
\hline 7 & 10 & 8 & $321(150-696)$ & 10 & 7 & $241(110-529)$ & 0 & 0 & $<2$ & 0 & 0 & $<2$ \\
\hline 8 & 1 & 0 & $2(1-14)$ & 2 & 0 & $5(1-16)$ & 0 & 0 & $<2$ & 1 & 0 & $2(1-14)$ \\
\hline 9 & 0 & 0 & $<2$ & 0 & 0 & $<2$ & 0 & 0 & $<2$ & 0 & 0 & $<2$ \\
\hline 10 & 1 & 0 & $2(1-14)$ & 1 & 0 & $2(1-14)$ & 0 & 0 & $<2$ & 0 & 0 & $<2$ \\
\hline 11 & 3 & 0 & $7(3-20)$ & 0 & 0 & $<2$ & 0 & 0 & $<2$ & 0 & 0 & $<2$ \\
\hline 12 & 7 & 0 & $21(10-43)$ & 4 & 1 & $12(5-28)$ & 0 & 0 & $<2$ & 0 & 0 & $<2$ \\
\hline 13 & 7 & 0 & $21(10-43)$ & 7 & 4 & $35(18-70)$ & 1 & 0 & $2(1-14)$ & 1 & 0 & $2(1-14)$ \\
\hline 14 & 10 & 7 & $241(110-529)$ & 10 & 10 & $>461$ & 0 & 0 & $<2$ & 1 & 1 & $4(1-16)$ \\
\hline 15 & 4 & 0 & $10(4-25)$ & 7 & 0 & $13(6-30)$ & 0 & 0 & $<2$ & 1 & 0 & $2(1-14)$ \\
\hline 16 & 2 & 0 & $5(1-16)$ & 5 & 0 & $13(6-30)$ & 0 & 0 & $<2$ & 0 & 0 & $<2$ \\
\hline 17 & 0 & 0 & $<2$ & 0 & 0 & $<2$ & 0 & 0 & $<2$ & 0 & 0 & $<2$ \\
\hline 18 & 4 & 1 & $12(5-28)$ & 5 & 0 & $13(6-30)$ & 0 & 0 & $<2$ & 0 & 0 & $<2$ \\
\hline 19 & 10 & 10 & $>461$ & 10 & 4 & $109(47-253)$ & 0 & 0 & $<2$ & 1 & 0 & $2(1-14)$ \\
\hline $20^{*}$ & & & & & & & 8 & 0 & $26(13-54)$ & 9 & 0 & $35(18-69)$ \\
\hline $21^{*}$ & & & & & & & 8 & 0 & $26(13-54)$ & 9 & 0 & $35(18-69)$ \\
\hline $22^{*}$ & & & & & & & 4 & 1 & $12(5-28)$ & 6 & 0 & $16(8-36)$ \\
\hline $23^{*}$ & & & & & & & 3 & 0 & $7(3-20)$ & 4 & 0 & $10(4-25)$ \\
\hline $24^{*}$ & & & & & & & 1 & 0 & $2(1-14)$ & 2 & 0 & $5(1-16)$ \\
\hline
\end{tabular}

FLA/L: Number of free-living amoebae per liter. Nf/L: Number of Naegleria fowleri per liter. * 20, 21, 22, 23, 24: Water doped with 36, 18, 8, 4, and 2 Naegleria fowleri per liter, respectively; number in the square brackets correspond to the $95 \%$ confidence limits with the lower and upper values. 


\subsection{Assessment of the Time and Storage Temperature of Water Samples, Prior to Processing}

To study the effect of temperature on FLA prior to water sample processing, we selected two temperatures: $4{ }^{\circ} \mathrm{C}$ (usually used for samples storage) [26] and $30{ }^{\circ} \mathrm{C}$, the average daily maximum air temperature in Guadeloupe. The results presented in Figure 1 show that the median numbers of thermophilic amoebae were not significantly different according to the temperature.

Regarding the time before sample processing, we selected several time windows ranging from $90 \mathrm{~min}$ to $2 \mathrm{~h}$ [26], 4 and $8 \mathrm{~h}$ (which is the maximum if the samples have to be processed on the same day as the sampling), and up to $24 \mathrm{~h}$ (in case water samples come from neighboring islands). Our results showed that there was no significant difference in the median number of thermophilic amoebae regardless of the delay between the collection and the culture.

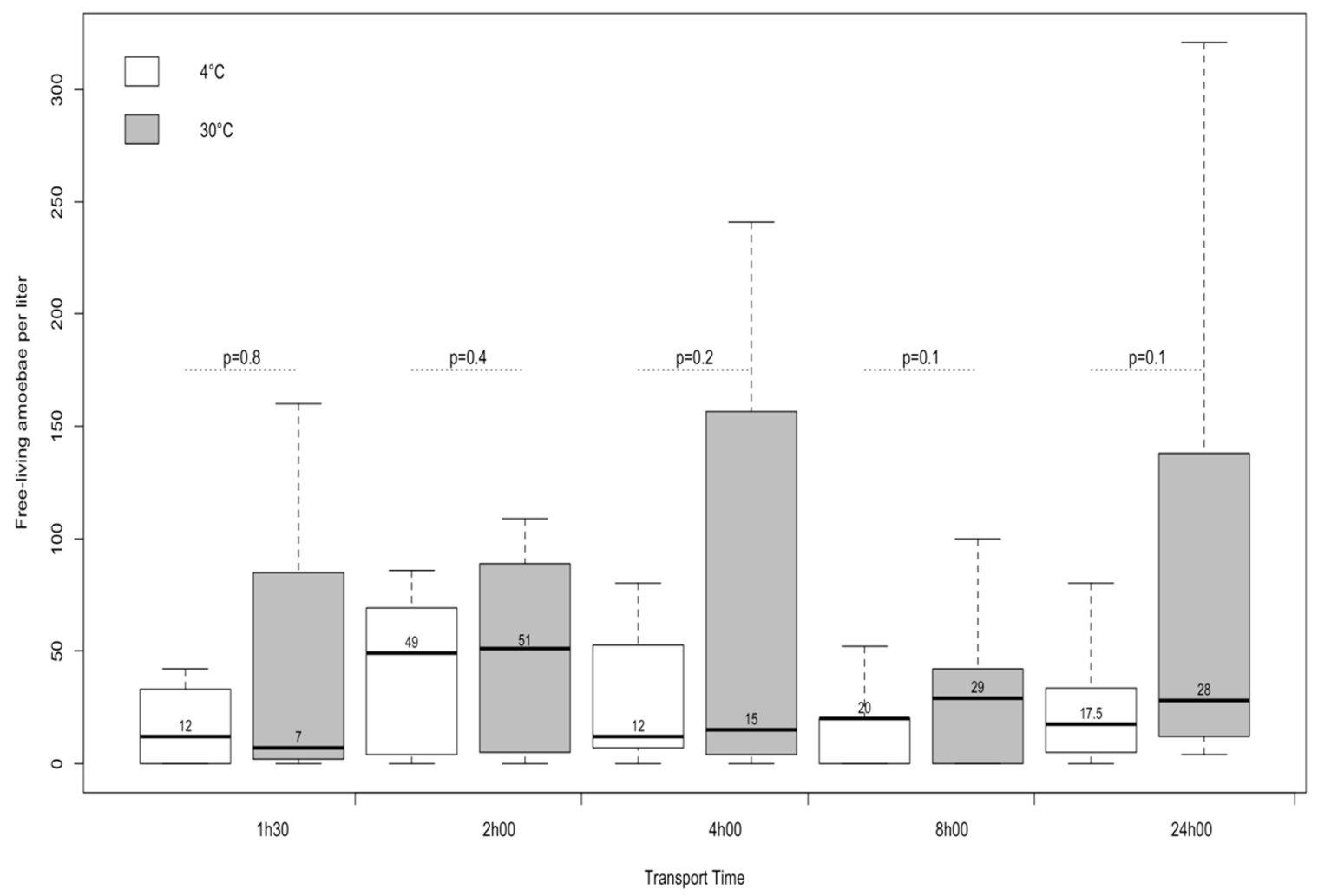

Figure 1. Median number of thermophilic amoebae per liter of water, at different times post-water sampling and at two storage temperatures $\left(+4^{\circ} \mathrm{C}\right.$ or $\left.+30^{\circ} \mathrm{C}\right)$ (box $1^{\circ}$ and $3^{\circ}$ quartile).

\section{Discussion}

Thermophilic FLA, and in particular Naegleria sp., have been frequently detected in Guadeloupe, in soil [11] and in natural thermal water [12]. French health authorities have endorsed a rule that forbids swimming or playing sports in natural water where the concentration of $N$. fowleri is above 100 amoebae/L [18]. As a preventive health measure, the local French health agency (ARS Guadeloupe) requests that we investigate 3-4 times per year the presence of Naegleria sp. (in particular N. fowleri) in the geothermal recreational waters often frequented by tourists and Guadeloupians. It is thus important to have an easy-to-use and reliable method to account for these FLA.

Many rapid detection assays have recently been developed and optimized to overcome conventional culture and microscopy techniques to detect $N$. fowleri in clinical and environmental samples. Many other studies also reported the superior sensitivity of real-time PCR over PCR, immunohistochemistry, or culture [16,17,21-25]. Herein, we used the MPN method because it allows identifying the number of viable, thermophilic, and cultivable FLA, such as Acanthamoeba, Naegleria, Vannella, and Vermamoeba. FLA viability can reflect the ability of trophozoites to multiply in water 
and the high resistance of FLA cysts to water treatments. Therefore, we can determine the potential dissemination of pathogenic FLA amoebae in habitats related to human population.

However, it is widely known that the classical MPN method (using either filtration or centrifugation to recover the FLA) is time-consuming, even if only performed with five replicates [27]. Our work clearly shows that this method can be substantially simplified. Indeed, our method using two samples (1000 and $100 \mathrm{~mL}$ ) filtered once is cheaper and much simpler than other methods, but most importantly, it is also reliable. Although we used a small number of water samples in our study, the new method gives results (FLA/L) similar to those obtained with the cMPN and enabled accounting for equal or more $N$. fowleri than the cMPN method. We believe that cutting a filter in 10 equal pieces instead of two increases the probability of amoebae getting in contact with $E$. coli seeded on the NNA, promoting their growth, and therefore reduces the competition between N. fowleri and other FLA for food [2]. This is particularly important when it comes to having a good estimate of $N$. fowleri concentrations in water.

This optimized method has an additional advantage: ease of sample transportation. With the mMPN method, we only need two sterile flasks per site, which can be transported at ambient temperature and can be processed within $24 \mathrm{~h}$ post-collection. These are very important features to take into account when it is necessary to perform water sampling in hot springs that are remote and difficult to access by car. Since natural thermal water baths are of small size in Guadeloupe (from 8 to $40 \mathrm{~m}^{2}$ ), this sampling is enough to give an indication of the contamination by N. fowleri.

Globally, our study demonstrates that this optimized method can replace the classical MPN method in any laboratory working on FLA. For instance, it is now routinely used in our laboratory to control Naegleria sp. in the water samples in Guadeloupe.

\section{Materials and Methods}

\subsection{Water Samples and Amoeba Isolation}

Two types of water samples were used: (i) those collected from 19 geothermal baths in Guadeloupe and (ii) five which were prepared with natural geothermal water that was filtered and then seeded with N. fowleri (previously isolated by our team from a hot spring in Guadeloupe) at different concentrations ranging from 2 to $36 \mathrm{~N}$. fowleri per liter (Table 1). All samples were pressure-filtered through a cellulose nitrate filter (pore size, $1.2 \mu \mathrm{m}$; diameter, $47 \mathrm{~mm}$, Millipore (FisherScientific)) [16,31].

\subsection{The Most Probable Number (MPN) Methods}

To compare the two counting methods, the water samples mentioned above were divided into two equal parts: one to be tested by the classical MPN method (cMPN) and the other with the modified MPN method (mMPN). The two methods are described below. All the plates for both procedures were incubated at $44^{\circ} \mathrm{C}$.

\subsubsection{Classical MPN Method}

For this, water samples of $1 \mathrm{~L}$ and $100 \mathrm{~mL}$ were filtered as follows: $10 \times 100$ and $10 \times 10 \mathrm{~mL}$, respectively $[17,26,27]$. Each filter was cut into two pieces, and placed inverted on a $1.5 \%$ non-nutrient agar plate seeded with a thin layer of Escherichia coli (NNA-E. coli), i.e., two pieces of filter per plate, per volume [17].

\subsubsection{Optimized MPN Method}

When $1 \mathrm{~L}$ and $100 \mathrm{~mL}$ water samples were collected, each sample was filtered only one time for each volume. Each filter was cut into 10 equal pieces (passing through the center of the filter), and placed inverted on a $1.5 \%$ NNA-E. coli plate, i.e., ten pieces of filter per plate, per volume. 


\subsection{Amoeba Identification and Counting}

During incubation, the first-line plates were examined daily macroscopically and microscopically using an inverted microscope. This allowed us to see the development of lytic areas over the bacterial coating, which corresponds to amoebic growth. Every amoeba plaque emerging along the filters was picked and subcultured on fresh NNA-E. coli (second-line plates). The isolates were pre-identified morphologically using Page's taxonomy key [32]. Amoeba identification was then performed by PCR using ITS and NFITS primers, as previously described [12,33].

For the cMPN method, the concentration of thermophilic FLA and N. fowleri was obtained by counting the total number of positive plates or each volume and by referring to the MPN table [34].

For the mMPN, the number of positive filter pieces was counted. Each filter piece obtained after filtering $100 \mathrm{~mL}$ was considered to be $10 \mathrm{~mL}$ of water, and each filter piece obtained after filtering $1 \mathrm{~L}$ was considered to be $100 \mathrm{~mL}$ of water. The numbers of positive piece of filter for each volume were reported in the same MPN statistical table.

\subsection{Assessment of Storage Temperature and Delay of Delivery of Water Samples}

To evaluate the impact of temperature storage and time on amoeba recovery, before filtration, we used eight water samples. Each sample was separated in 10 parts: Five were kept at ambient temperature $\left(+30^{\circ} \mathrm{C}\right)$, and the others were stored at $+4{ }^{\circ} \mathrm{C}$. At different times post-collection, one sample that was stored at ambient temperature and one that was stored at $+4{ }^{\circ} \mathrm{C}$ were filtered and cultured. For this, we used the classical MPN method by filtering $1100 \mathrm{~mL}$ of water as described above.

\subsection{Statistical Analyses}

All statistical analyses were performed using R software, version 3.5.2 (R Foundation for Statistical Computing, Vienna, Austria. URL https://www.R-project.org/). The means of positives cultures and the estimated numbers of colonies obtained after new vs. classical MPN methods, and mean numbers of colonies obtained after transportation at +4 vs. $+30{ }^{\circ} \mathrm{C}$ were compared using the nonparametric Wilcoxon rank test for paired series. The mean numbers of colonies that were obtained with a transportation delay were compared using the Kruskal-Wallis nonparametric test. For all comparisons, $p$-values $<0.05$ were considered to be statistically significant.

Author Contributions: Conceptualization and methodology, A.T., M.M., and J.G.; formal analysis, A.T., M.M., J.G., and V.R.; investigation, M.M., writing—original draft preparation, A.T. and M.M.; writing-review and editing, A.T., J.G., M.M., and I.M.; All authors have read and agreed to the published version of the manuscript.

Funding: This work was supported by a grant ACIP $n^{\circ}$ A01-11 from the Pasteur Institut and by a grant FEDER FED-1-1.4 32932 from the European Union. Mirna Moussa was supported by a grant FSE from the European Union and the Guadeloupe Region.

Conflicts of Interest: The authors declare no conflict of interest. The funders had no role in the design of the study; in the collection, analyses, or interpretation of data; in the writing of the manuscript, or in the decision to publish the results.

\section{References}

1. Adamska, M.; Leonska-Duniec, A.; Lanocha, N.; Skotarczak, B. Thermophilic potentially pathogenic amoebae isolated from natural water bodies in Poland and their molecular characterization. Acta Parasitol. 2014, 59, 433-441. [CrossRef] [PubMed]

2. Tyndall, R.L.; Ironside, K.S.; Metler, P.L.; Tan, E.L.; Hazen, T.C.; Fliermans, C.B. Effect of thermal additions on the density and distribution of thermophilic amoebae and pathogenic Naegleria fowleri in a newly created cooling lake. Appl. Environ. Microbiol. 1989, 55, 722-732. [CrossRef] [PubMed]

3. Scheid, P. Free-Living Amoebae as Human Parasites and Hosts for Pathogenic Microorganisms. Proceedings 2018, 2, 692. [CrossRef] 
4. Latifi, A.R.; Niyyati, M.; Lorenzo-Morales, J.; Haghighi, A.; Seyyed Tabaei, S.J.; Lasjerdi, Z. Presence of Balamuthia mandrillaris in hot springs from Mazandaran province, northern Iran. Epidemiol. Infect. 2016, 144, 2456-2461. [CrossRef] [PubMed]

5. Visvesvara, G.S.; Moura, H.; Schuster, F.L. Pathogenic and opportunistic free-living amoebae: Acanthamoeba spp., Balamuthia mandrillaris, Naegleria fowleri, and Sappinia diploidea. FEMS Immunol. Med. Microbiol. 2007, 50, 1-26. [CrossRef] [PubMed]

6. Niyyati, M.; Latifi, A. Free Living Amoeba Belonging to Vannella Spp. Isolated from a Hotspring in Amol City, Northern Iran. Nov. Biomed. 2017, 5, 85-88.

7. Delafont, V.; Rodier, M.-H.; Maisonneuve, E.; Cateau, E. Vermamoeba vermiformis: A Free-Living Amoeba of Interest. Microb. Ecol. 2018, 76, 991-1001. [CrossRef]

8. Walochnik, J. Amoebae. In Parasitic Protozoa of Farm Animals and Pets; Springer International Publishing: Cham, Switzerland, 2018; pp. 389-412, ISBN 9783319701325.

9. Scheid, P.L. Vermamoeba vermiformis-A Free-Living Amoeba with Public Health and Environmental Health Significance. Open Parasitol. J. 2019, 7, 40-47. [CrossRef]

10. Scheid, P.L.; Lâm, T.-T.; Sinsch, U.; Balczun, C. Vermamoeba vermiformis as etiological agent of a painful ulcer close to the eye. Parasitol. Res. 2019, 118, 1999-2004. [CrossRef]

11. Moussa, M.; Tissot, O.; Guerlotté, J.; De Jonckheere, J.F.; Talarmin, A. Soil is the origin for the presence of Naegleria fowleri in the thermal recreational waters. Parasitol. Res. 2015, 114, 311-315. [CrossRef]

12. Moussa, M.; De Jonckheere, J.F.; Guerlotté, J.; Richard, V.; Bastaraud, A.; Romana, M.; Talarmin, A. Survey of Naegleria fowleri in Geothermal Recreational Waters of Guadeloupe (French West Indies). PLoS ONE 2013, 8, e54414. [CrossRef]

13. Cárdenas-Zúñiga, R.; Martínez-Castillo, M.; Shibayama, M.; Serrano-Luna, J.; Debnath, A.; Coronado-Velázquez, D. Naegleria fowleri after 50 years: Is it a neglected pathogen? J. Med. Microbiol. 2016, 65, 885-896.

14. Siddiqui, R.; Ali, I.K.M.; Cope, J.R.; Khan, N.A. Biology and pathogenesis of Naegleria fowleri. Acta Trop. 2016, 164, 375-394. [CrossRef] [PubMed]

15. Nicolas, M.; De Jonckheere, J.F.; Pernin, P.; Bataille, H.; Le Bris, V.; Herrmann-Storck, C. Molecular diagnosis of a fatal primary amoebic meningoencephalitis in Guadeloupe (French West Indies). Bull. Soc. Pathol. Exot. 2010, 103, 14-18. [CrossRef] [PubMed]

16. Behets, J.; Declerck, P.; Delaedt, Y.; Verelst, L.; Ollevier, F. A duplex real-time PCR assay for the quantitative detection of Naegleria fowleri in water samples. Water Res. 2007, 41, 118-126. [CrossRef] [PubMed]

17. Pernin, P.; Pélandakis, M.; Rouby, Y.; Faure, A.; Siclet, F. Comparative recoveries of Naegleria fowleri amoebae from seeded river water by filtration and centrifugation. Appl. Environ. Microbiol. 1998, 64, 955-959. [CrossRef] [PubMed]

18. Cabanes, P.A.; Wallet, F.; Pringuez, E.; Pernin, P. Assessing the Risk of Primary Amoebic Meningoencephalitis from Swimming in the Presence of Environmental Naegleria fowleri. Appl. Environ. Microbiol. 2001, 67, 2927-2931. [CrossRef]

19. Percival, S.; Chalmers, R.; Embrey, M.; Hunter, P.; Sellwood, J.; Wyn-Jones, P. Naegleria fowleri. Microbiol. Waterborne Dis. 2004, 35, 319-324.

20. Maciver, S.K.; Piñero, J.E.; Lorenzo-Morales, J. Is Naegleria fowleri an Emerging Parasite? Trends Parasitol. 2020, 36, 19-28. [CrossRef]

21. Mahittikorn, A.; Mori, H.; Popruk, S.; Roobthaisong, A.; Sutthikornchai, C.; Koompapong, K.; Siri, S.; Sukthana, Y.; Nacapunchai, D. Development of a Rapid, Simple Method for Detecting Naegleria fowleri Visually in Water Samples by Loop-Mediated Isothermal Amplification (LAMP). PLoS ONE 2015, 10, e0120997. [CrossRef]

22. Le Calvez, T.; Trouilhé, M.-C.; Humeau, P.; Moletta-Denat, M.; Frère, J.; Héchard, Y. Detection of free-living amoebae by using multiplex quantitative PCR. Mol. Cell. Probes 2012, 26, 116-120. [CrossRef] [PubMed]

23. Flores, B.M.; Garcia, C.A.; Stamm, W.E.; Torian, B.E. Differentiation of Naegleria fowleri from Acanthamoeba species by using monoclonal antibodies and flow cytometry. J. Clin. Microbiol. 1990, 28, 1999-2005. [CrossRef] [PubMed]

24. Johnson, P.E.; Deromedi, A.J.; Lebaron, P.; Catala, P.; Havens, C.; Pougnard, C. High throughput, real-time detection of Naegleria lovaniensis in natural river water using LED-illuminated Fountain Flow Cytometry. J. Appl. Microbiol. 2007, 103, 700-710. [CrossRef] [PubMed] 
25. Pougnard, C.; Catala, P.; Drocourt, J.L.; Legastelois, S.; Pernin, P.; Pringuez, E.; Lebaron, P. Rapid detection and enumeration of Naegleria fowleri in surface waters by solid-phase cytometry. Appl. Environ. Microbiol. 2002, 68, 3102-3107. [CrossRef] [PubMed]

26. International Organization for Standardization. International Organization for Standardization: ISO 8199:2005. Water Quality—General Guidance on the Enumeration of Micro-Organisms by Culture; International Organization for Standardization: Geneva, Switzerland, 2005.

27. Lares-Villa, F.; Hernández-Peña, C. Concentration of Naegleria fowleri in natural waters used for recreational purposes in Sonora, Mexico (November 2007-October 2008). Exp. Parasitol. 2010, 126, 33-36. [CrossRef]

28. Beattie, T.K.; Seal, D.V.; Tomlinson, A.; McFadyen, A.K.; Grimason, A.M. Determination of Amoebicidal Activities of Multipurpose Contact Lens Solutions by Using a Most Probable Number Enumeration Technique. J. Clin. Microbiol. 2003, 41, 2992-3000. [CrossRef]

29. Dietersdorfer, E.; Cervero-Aragó, S.; Sommer, R.; Kirschner, A.K.; Walochnik, J. Optimized methods for Legionella pneumophila release from its Acanthamoeba hosts. BMC Microbiol. 2016, 16, 74. [CrossRef]

30. Page, F.C. A revised classification of the Gymnamoebia (Protozoa: Sarcodina). Zool. J. Linn. Soc. 1976, 61-77. [CrossRef]

31. Edagawa, A.; Kimura, A.; Kawabuchi-Kurata, T.; Kusuhara, Y.; Karanis, P. Isolation and genotyping of potentially pathogenic Acanthamoeba and Naegleria species from tap-water sources in Osaka, Japan. Parasitol. Res. 2009, 105, 1109-1117. [CrossRef]

32. Page, F.C. A New Key to Freshwater and Soil Gymnamoebae with Instruction for Culture; Freshwater Biological Association: Ambleside, UK, 1988; 122p.

33. De Jonckheere, J.F. Sequence Variation in the Ribosomal Internal Transcribed Spacers, Including the $5.8 \mathrm{~S}$ rDNA, of Naegleria spp. Protist 1998, 149, 221-228. [CrossRef]

34. Champsaur, H. Méthodes générales d'examen bactériologique des eaux. In L'analyse de L'eau; Rodier, J., Ed.; Dunod: Paris, France, 1996; pp. 755-756.

(C) 2020 by the authors. Licensee MDPI, Basel, Switzerland. This article is an open access article distributed under the terms and conditions of the Creative Commons Attribution (CC BY) license (http://creativecommons.org/licenses/by/4.0/). 